\title{
Prediction of VLE of polymer solutions by the GFD equation of state
}

\author{
Jung-Chin Tsai, Muoi Tang ${ }^{1}$, Yan-Ping Chen * \\ Department of Chemical Engineering, National Taiwan University, Taipei, Taiwan
}

Received 12 April 1998; accepted 26 January 1999

\begin{abstract}
The generalized-Flory dimer (GFD) equation of state (EOS) is combined with the UNIFAC-FV group contribution $G^{\mathrm{E}}$ model to yield predictive vapor-liquid equilibrium calculations of polymer solutions. The equilibrium pressures, solvent solubility and activity of various binary systems are calculated. The GFD $/ G^{\mathrm{E}}$ method predicts good results in comparison to those from other optimally fitted models. (C) 1999 Elsevier Science B.V. All rights reserved.
\end{abstract}

Keywords: Equation of state; Method of calculation; VLE; Polymer solution

\section{Introduction}

Equation of state (EOS) methods are commonly use in thermodynamic and phase equilibrium calculations. Recently, some theoretically-based EOS have been presented, for example the SAFT [1] and the GFD [2] EOS. Both EOS are capable of representing the properties of a variety of substances ranging from small molecular weight to chain molecules. Applications of the GFD EOS to real fluid systems up to high polymers have been described in previous studies $[3,4]$ with satisfactory results. It is of interest to employ the group contribution mixing rules with cubic or non-cubic type EOS $[5,6]$ for chemical process design. The objective of this work is to predict the VLE of polymer solutions using the GFD EOS and the UNIFAC-FV $G^{\mathrm{E}}$ model, and to compare the results with those from other correlative methods.

\footnotetext{
${ }^{*}$ Corresponding author. Tel.: + 886-2236-61661; fax: + 886-2236-23040; e-mail: ypchen@ccms.ntu.edu.tw

${ }^{1}$ Present address: Department of Chemical Engineering, Chinese Culture University, Taipei, Taiwan.
} 


\section{Methods of calculation}

The GFD EOS is written in the following form:

$$
Z_{m}=Z_{\mathrm{I}}+\left[\left(V_{\mathrm{e}, m}-V_{\mathrm{e}, \mathrm{I}}\right) /\left(V_{\mathrm{e}, \mathrm{II}}-V_{\mathrm{e}, \mathrm{I}}\right)\right]\left(Z_{\mathrm{II}}-Z_{\mathrm{I}}\right)
$$

where $Z$ is the compressibility factor and $V_{\mathrm{e}}$ is the excluded volume. The subscripts I, II and $m$ denote a monomer, dimer and $m$-mer fluid, respectively. The EOS shown in Eq. (1) is a function of three parameters: the number of segments $m$, the molar volume of a hard sphere segment $V^{0}$, and the interaction energy parameter $\varepsilon / k T$. Wu and Chen [3] have expressed the volume and energy parameters as functions of temperature that gives satisfactory results in correlating the saturated properties of pure fluids:

$$
\begin{aligned}
& V^{0}=V^{00}+0.005 T \\
& \varepsilon / k T=\varepsilon^{0} / k T-0.04 T
\end{aligned}
$$

The parameters $m, V^{00}$ and $\varepsilon^{0} / k T$ have been regressed by $\mathrm{Wu}$ and Chen [3] for pure low molecular weight compounds and polymers. For the VLE calculation on polymer solutions, the equal fugacity criterion applies:

$$
f_{1}^{\mathrm{V}}=\hat{f}_{1}^{\mathrm{L}}
$$

where the subscript 1 represents the solvent molecule and no polymer is assumed to exist in the vapor phase. For the segment number and the volume parameters, simple van der Waals type mixing rules are used in fluid mixture calculations:

$$
\begin{aligned}
& m=\sum x_{i} m_{i} \\
& m V^{0}=\sum x_{i}\left(m_{i} V_{i}^{0}\right)
\end{aligned}
$$

The interaction energy parameter of a mixture can be determined in a correlative form from traditional mixing rules and a binary parameter $k_{i j}$ :

$$
\varepsilon / k T=\sum \sum s_{i} s_{j}\left(\varepsilon_{i} \varepsilon_{j}\right)^{0.5}\left(1-k_{i j}\right) / k T
$$

where $s$ is the segment fraction. An alternative $\operatorname{EOS} / G^{\mathrm{E}}$ mixing rule, which is a modification of the previous work from Huron and Vidal [7], can be applied to the GFD EOS. In this case, the excess Gibbs free energy from the GFD EOS at the system's temperature and pressure is set equal to that from a $G^{\mathrm{E}}$ model:

$$
\left(G^{\mathrm{E}} / R T\right)_{P}^{\mathrm{EOS}}=\left(G^{\mathrm{E}} / R T\right)^{G^{\mathrm{E}} \text { model }}
$$

Using the group contribution UNIFAC-FV model [8], the GFD EOS energy parameter of a mixture is calculated from Eqs. (1) and (8). With this energy parameter and the mixture parameters from Eqs. (5) and (6), VLE calculations of polymer solutions are carried out by solving Eq. (4).

\section{Results and discussion}

Table 1 lists the calculated results of the solubilities of solvents in polymers. We compare our predicted results with those from various EOS methods [1,9] using traditional mixing rules and their 
Table 1

Calculated solubilities of solvents in polymers from the various EOS

\begin{tabular}{|c|c|c|c|c|c|c|c|c|c|c|}
\hline \multirow{2}{*}{$\begin{array}{l}\text { System, solvent(1) } \\
+ \text { polymer }(2)\end{array}$} & \multirow{2}{*}{$\begin{array}{l}\text { Temperature } \\
\text { range }(\mathrm{K})\end{array}$} & \multirow{2}{*}{$\begin{array}{l}\text { Number of } \\
\text { data points }\end{array}$} & \multicolumn{2}{|l|}{ SL EOS } & \multicolumn{2}{|l|}{ GFD } & \multicolumn{2}{|l|}{ SAFT } & \multirow{2}{*}{$\begin{array}{l}\text { This work, } \\
\text { Dev } w_{1}\end{array}$} & \multirow{2}{*}{$\begin{array}{l}\text { Data } \\
\text { sources }\end{array}$} \\
\hline & & & Optimum $k_{12}$ & Dev $w_{1}$ & Optimum $k_{12}$ & $\operatorname{Dev} w_{1}$ & Optimum $k_{12}$ & $\operatorname{Dev} w_{1}$ & & \\
\hline Hexane + PE & $383-473$ & 100 & 0.01395 & 0.0183 & -0.04321 & 0.0221 & -0.00657 & 0.0313 & 0.0115 & [11] \\
\hline Heptane + PE & $383-473$ & 100 & 0.00389 & 0.0144 & -0.03239 & 0.0196 & -0.00515 & 0.0288 & 0.0157 & [11] \\
\hline Benzene $+\mathrm{PE}$ & $383-473$ & 100 & 0.00384 & 0.0112 & -0.00457 & 0.0182 & 0.00692 & 0.0299 & 0.0149 & [11] \\
\hline Toluene $+\mathrm{PE}$ & $383-473$ & 100 & 0.00042 & 0.0147 & -0.01114 & 0.0115 & 0.00062 & 0.0155 & 0.0129 & [11] \\
\hline Cyclohexane + PE & $383-473$ & 100 & -0.00912 & 0.0154 & -0.03367 & 0.0138 & -0.00515 & 0.0385 & 0.0214 & [11] \\
\hline Ethylbenzene + PS & $393-458$ & 55 & 0.00803 & 0.0023 & -0.09269 & 0.0051 & 0.01811 & 0.0261 & 0.0030 & [12] \\
\hline Methylethylketone + PS & $298-343$ & 16 & 0.01257 & 0.0204 & -0.08079 & 0.0163 & -0.32052 & 0.0262 & 0.0164 & [13] \\
\hline Benzene + PS & $293-393$ & 38 & 0.00168 & 0.0110 & -0.11398 & 0.0288 & -0.35745 & 0.0325 & 0.0171 & [14] \\
\hline Toluene + PS & $293-333$ & 35 & -0.00213 & 0.0217 & -0.10130 & 0.0212 & -0.31959 & 0.0342 & 0.0161 & {$[13,14]$} \\
\hline$m$-Xylene + PS & $403-448$ & 22 & 0.00703 & 0.0021 & -0.08023 & 0.0041 & -0.21003 & 0.0104 & 0.0031 & [15] \\
\hline Butane + PIB & $308-319$ & 10 & 0.02065 & 0.0108 & -0.14160 & 0.0215 & -0.05042 & 0.0010 & 0.0158 & [16] \\
\hline Pentane + PIB & $318-328$ & 10 & 0.00845 & 0.0178 & 0.02832 & 0.0860 & 0.07872 & 0.0910 & 0.0097 & [16] \\
\hline Benzene + PVAC & 303 & 8 & 0.01071 & 0.0124 & -0.04998 & 0.0098 & -0.02718 & 0.0269 & 0.0170 & [17] \\
\hline Ethyl benzene + BR & $353-403$ & 35 & 0.00982 & 0.0196 & -0.04001 & 0.0165 & - & - & 0.0131 & [16] \\
\hline 3-Pentanone + PP & $298-318$ & 22 & 0.03061 & 0.0115 & 0.09821 & 0.0177 & 0.03201 & 0.0103 & 0.0396 & [18] \\
\hline Grand average & & 751 & & 0.0138 & & 0.0175 & & 0.0283 & 0.0147 & \\
\hline
\end{tabular}

PE: Polyethylene, PS: Polystyrene, PIB: Polyisobtylene, PVAC: Polyvinyl acetate, PP: Polypropylene, BR: $c i s-1,4-$ Polybutadiene; Dev $w_{1}=1 / N \sum \mid w_{1}^{\exp }-$ $w_{1}^{\text {cal }}$. 


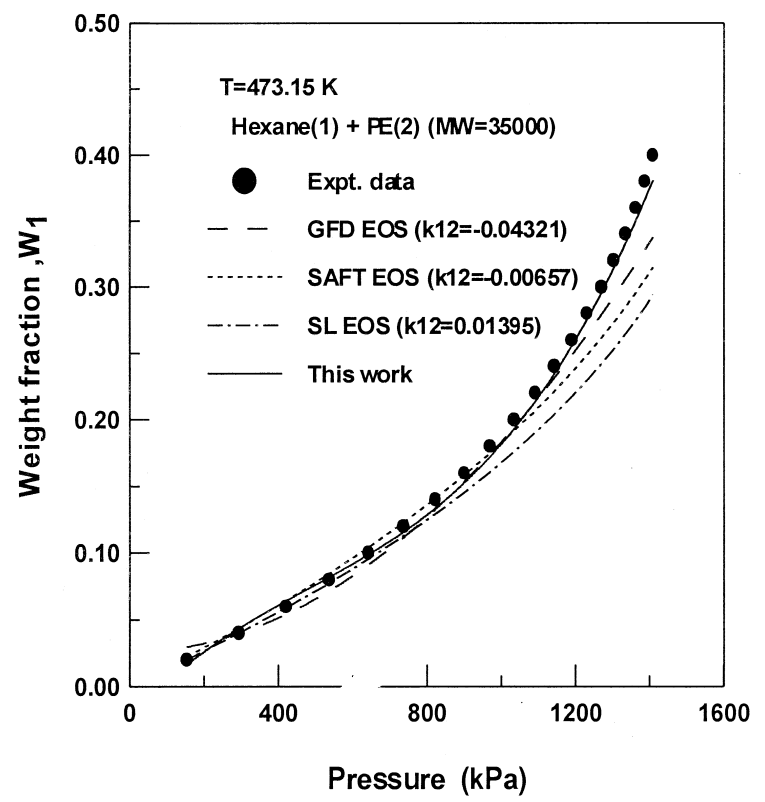

Fig. 1. Comparison of the calculated solubilities of hexane in polyethylene from various equations of state.

optimally fitted binary parameters. The predictive results from the GFD $/ G^{\mathrm{E}}$ method are of the same accuracy as that from the best fitted methods. Fig. 1 shows the predicted solubilities of hexane in polyethylene which agree well with experimental data. Calculated results for the equilibrium pressures of polymer solutions from various EOS methods are presented in Table 2. The GFD EOS with group contribution mixing rules also shows satisfactory accuracy. The maximum calculated deviation from this work is smaller than that from other methods. Graphical presentations of the equilibrium pressure calculations are shown in Figs. 2 and 3. Table 3 shows the calculated activity of solvents in polymer solutions from various approaches. The GFD EOS with predictive mixing rules demonstrates comparable accuracy to the SAFT EOS with its best fitted binary parameters. The predictive calculations from the GFD EOS again show less peak deviations from the experimental data than the other correlations. Table 3 also shows the comparison of the results from this study with those from the other two group contribution methods of the GC-Flory [10] and the UNIFAC-FV equations. The GFD EOS with group contribution mixing model shows superior results to the other two predictive models. Graphical comparisons of the calculated activity are shown in Fig. 4. The non-cubic GFD EOS is successfully employed in predictive VLE calculations of polymer solutions. The results from the GFD $/ G^{\mathrm{E}}$ method show satisfactory accuracy.

\section{Conclusion}

The GFD EOS is combined with the UNIFAC-FV activity coefficient model to predict VLE of polymer solutions. The equilibrium pressure, solvent solubility and activity of various polymer 
Table 2

Results of vapor-liquid equilibrium calculations on polymer solutions using various EOS

\begin{tabular}{|c|c|c|c|c|c|c|c|c|c|c|}
\hline \multirow{2}{*}{$\begin{array}{l}\text { System, solvent(1) } \\
+ \text { polymer(2) }\end{array}$} & \multirow{2}{*}{$\begin{array}{l}\text { Temperature } \\
\text { range }(\mathrm{K})\end{array}$} & \multirow{2}{*}{$\begin{array}{l}\text { Number of } \\
\text { data points }\end{array}$} & \multicolumn{2}{|l|}{ SL EOS } & \multicolumn{2}{|l|}{ GFD } & \multicolumn{2}{|l|}{ SAFT } & \multirow{2}{*}{$\begin{array}{l}\text { This work, } \\
\text { AAD } P(\%)\end{array}$} & \multirow{2}{*}{$\begin{array}{l}\text { Data } \\
\text { sources }\end{array}$} \\
\hline & & & Optimum $k_{12}$ & AAD $P(\%)$ & Optimum $k_{12}$ & AAD $P(\%)$ & Optimum $k_{12}$ & AAD $P(\%)$ & & \\
\hline Hexane + PE & $383-473$ & 100 & 0.01376 & 5.32 & -0.04116 & 6.40 & -0.00682 & 2.41 & 3.89 & [11] \\
\hline Heptane + PE & $383-473$ & 100 & 0.00508 & 5.95 & -0.03530 & 5.82 & -0.00668 & 2.19 & 8.22 & [11] \\
\hline Benzene $+\mathrm{PE}$ & $383-473$ & 100 & 0.00409 & 5.36 & -0.01108 & 5.75 & 0.00529 & 2.13 & 8.59 & [11] \\
\hline Toluene + PE & $383-473$ & 100 & 0.00222 & 3.74 & -0.01363 & 3.98 & -0.00121 & 1.51 & 6.15 & [11] \\
\hline Ethylbenzene + PS & $393-458$ & 55 & 0.00513 & 5.71 & -0.08508 & 14.25 & -0.02240 & 16.68 & 5.96 & [12] \\
\hline Methylethylketone + PS & $298-343$ & 16 & 0.01409 & 5.42 & -0.08078 & 3.26 & -0.19516 & 8.07 & 3.57 & [13] \\
\hline Benzene + PS & $293-393$ & 38 & 0.00409 & 7.03 & -0.08372 & 8.77 & 0.01621 & 10.83 & 10.35 & [14] \\
\hline Toluene + PS & $293-333$ & 35 & -0.00185 & 14.45 & -0.08421 & 11.93 & -0.25639 & 17.21 & 9.23 & {$[13,14]$} \\
\hline$m$-Xylene + PS & $403-448$ & 22 & 0.00698 & 6.76 & -0.07952 & 6.62 & -0.19484 & 19.02 & 5.12 & [15] \\
\hline Cyclohexane + PS & $297-307$ & 17 & 0.02921 & 6.71 & 0.01371 & 5.46 & 0.04305 & 3.46 & 5.85 & [19] \\
\hline Hexane + PIB & 398 & 20 & 0.01513 & 6.03 & -0.06730 & 4.06 & -0.02613 & 4.23 & 9.88 & [11] \\
\hline Cyclohexane + PIB & 398 & 20 & 0.01166 & 4.88 & -0.04284 & 4.52 & -0.01757 & 1.64 & 9.12 & [11] \\
\hline Benzene + PVAC & 303 & 8 & 0.01041 & 3.48 & -0.04960 & 4.41 & -0.02803 & 4.08 & 3.94 & [17] \\
\hline Ethyl benzene $+B R$ & $353-403$ & 35 & 0.00818 & 9.30 & -0.04368 & 10.22 & - & - & 9.56 & [16] \\
\hline Hexane + PP & 353 & 10 & 0.00367 & 4.46 & 0.11145 & 1.57 & -0.00133 & 19.55 & 3.79 & [18] \\
\hline Grand average & & 676 & & 6.05 & & 6.81 & & 5.78 & 7.07 & \\
\hline
\end{tabular}

$\operatorname{AAD} P(\%)=100 / N \Sigma\left|P^{\exp }-P^{\text {cal }}\right| / P^{\exp }$ 


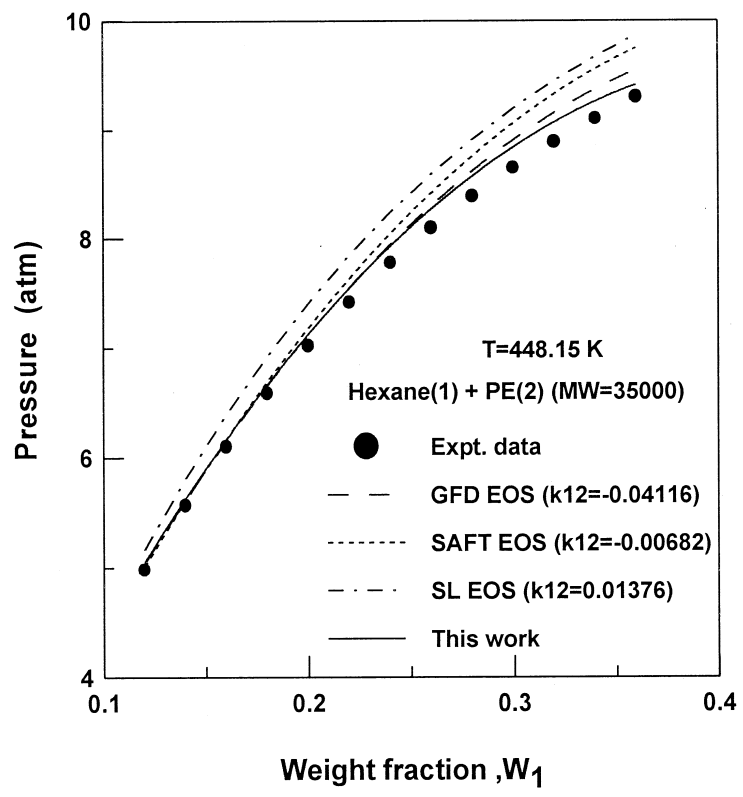

Fig. 2. Comparison of the calculated VLE results for hexane in polyethylene from various equations of state.

solution systems are calculated with satisfactory accuracy. The non-cubic GFD EOS with predictive mixing rules shows good results in comparison to those from other optimally fitted approaches.

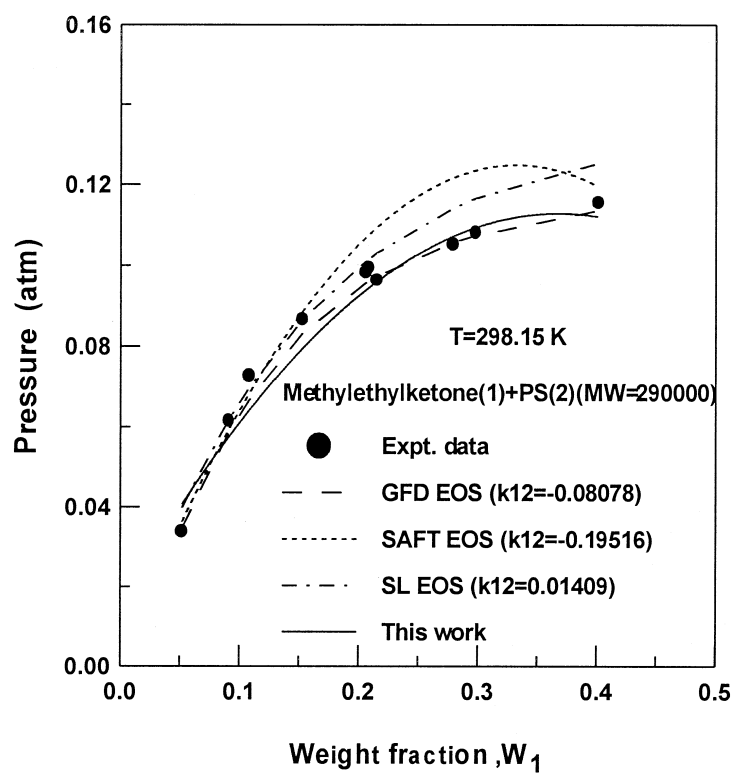

Fig. 3. Comparison of the calculated VLE results for methyl ethyl ketone in polystyrene from various equations of state. 
Table 3

Comparison of calculated activity deviation for polymer solutions from various thermodynamic models

\begin{tabular}{|c|c|c|c|c|c|c|c|c|}
\hline \multirow{2}{*}{$\begin{array}{l}\text { System, solvent(1) } \\
+\operatorname{polymer}(2)\end{array}$} & \multirow{2}{*}{$\begin{array}{l}\text { Temperature } \\
\text { range }(\mathrm{K})\end{array}$} & \multirow{2}{*}{$\begin{array}{l}\text { Number of } \\
\text { data points }\end{array}$} & \multirow{2}{*}{$\begin{array}{l}\text { GC-Flory } \\
\text { AAD } a_{1}(\%)\end{array}$} & \multirow{2}{*}{$\begin{array}{l}\text { UNIFAC-FV } \\
\text { AAD } a_{1}(\%)\end{array}$} & \multicolumn{2}{|l|}{ SAFT } & \multirow{2}{*}{$\begin{array}{l}\text { This work, } \\
\text { AAD } a_{1}(\%)\end{array}$} & \multirow{2}{*}{$\begin{array}{l}\text { Data } \\
\text { sources }\end{array}$} \\
\hline & & & & & Optimum $k_{12}$ & $\operatorname{AAD} a_{1}(\%)$ & & \\
\hline Hexane + PE & $383-473$ & 100 & 6.32 & 15.78 & -0.00795 & 6.91 & 2.92 & [11] \\
\hline Heptane + PE & $383-473$ & 100 & 7.43 & 14.81 & -0.00639 & 5.56 & 5.93 & [11] \\
\hline Octane + PE & $383-473$ & 100 & 7.47 & 14.19 & -0.00496 & 4.10 & 5.57 & [11] \\
\hline Cyclohexane + PE & $383-473$ & 100 & 8.24 & 10.57 & -0.00620 & 4.52 & 7.42 & [11] \\
\hline Toluene + PE & $383-473$ & 100 & 18.53 & 4.11 & -0.00039 & 2.87 & 6.64 & [11] \\
\hline Ethylbenzene + PS & $393-458$ & 55 & 6.69 & 12.99 & 0.01697 & 5.28 & 5.46 & {$[12]$} \\
\hline Methylethylketone + PS & $298-343$ & 16 & 16.22 & 12.68 & -0.19551 & 9.35 & 3.30 & [13] \\
\hline Benzene + PS & $293-393$ & 38 & 20.47 & 10.48 & 0.02634 & 2.45 & 7.93 & {$[14]$} \\
\hline Toluene + PS & $293-333$ & 35 & 21.19 & 16.37 & -0.25088 & 17.47 & 9.52 & {$[13,14]$} \\
\hline Cyclohexane + PS & $297-317$ & 54 & 19.69 & 13.75 & 0.04341 & 10.24 & 8.26 & [19] \\
\hline Hexane + PIB & $383-473$ & 100 & 39.78 & 13.01 & -0.02562 & 9.50 & 10.97 & {$[11]$} \\
\hline Cyclohexane + PIB & $383-473$ & 100 & 47.16 & 8.59 & -0.01532 & 5.16 & 8.65 & [11] \\
\hline Pentane + PIB & $318-328$ & 10 & 23.84 & 2.88 & 0.07146 & 8.07 & 4.88 & {$[16]$} \\
\hline Benzene + PVAC & 303 & 8 & 11.24 & 3.66 & -0.02911 & 4.07 & 9.13 & {$[17]$} \\
\hline Hexane + PP & 353 & 10 & 10.32 & 8.26 & 0.01174 & 4.39 & 4.11 & [18] \\
\hline Grand average & & 926 & 18.23 & 11.75 & & 6.17 & 6.92 & \\
\hline
\end{tabular}

$\operatorname{AAD} a_{1}(\%)=100 / N \Sigma\left|a_{1}^{\text {exp }}-a_{1}^{\text {cal }}\right| / a_{1}^{\exp }$. 


\section{List of symbols}

$\begin{array}{ll}f & \text { fugacity } \\ G & \text { Gibbs free energy } \\ k & \text { Boltzmann constant, or binary interaction parameter } \\ m & \text { segment number } \\ P & \text { pressure } \\ R & \text { gas constant } \\ S & \text { segment fraction } \\ T & \text { temperature } \\ V & \text { volume } \\ w & \text { weight fraction } \\ x & \text { mole fraction } \\ Z & \text { compressibility factor }\end{array}$

Greek letter

$\varepsilon \quad$ energy parameter of the GFD EOS

$\phi \quad$ fugacity coefficient

Subscripts

$m \quad m$-mer fluid

I, II monomer and dimer

1,2 components 1 and 2

$i, j, k \quad$ components $i, j$ and $k$

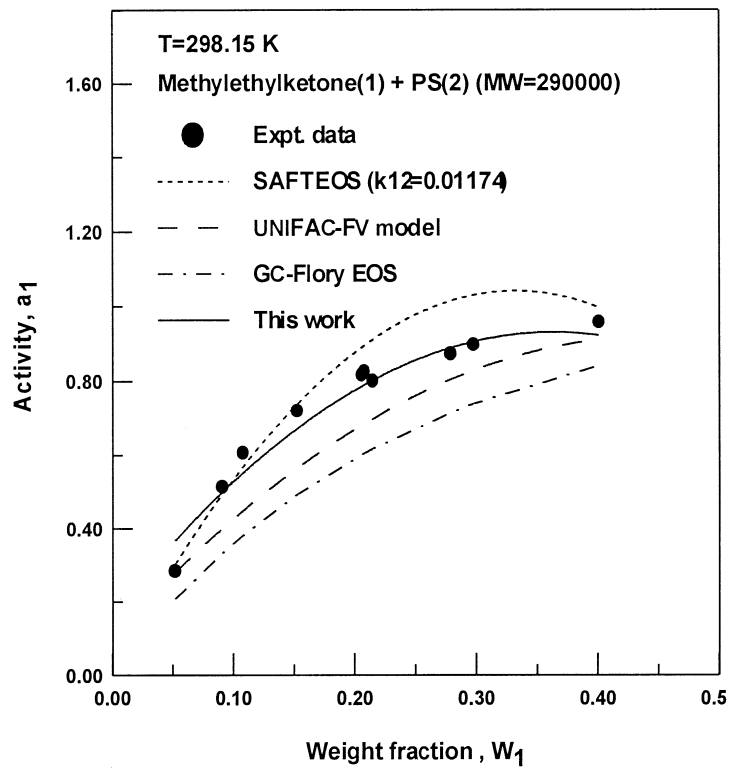

Fig. 4. Comparison of the calculated activity of methyl ethyl ketone in polystyrene from various thermodynamic models. 


\section{Superscripts}

$\mathrm{E}$ excess property

L liquid phase

$\mathrm{V} \quad$ vapor phase

\section{Appendix A}

The excess Gibbs free energy is calculated from the GFD EOS:

$$
\left(G^{\mathrm{E}} / R T\right)_{P}^{\mathrm{EOS}}=\sum x_{i} \ln \left(\hat{\phi}_{i} / \phi_{i}\right)
$$

The fugacity coefficient of a pure component $i$ calculated from the GDF EOS is:

$$
\ln \phi_{i}=-\left(Y_{m}+1\right) B_{2}+Y_{m} B_{1}-\ln Z+Z-1
$$

where

$$
\begin{aligned}
Y_{m}= & -2.08496+1.12743 m-0.04248 m^{2} \text { for } m \leq 2 \\
Y_{m}= & (m-2) \times 0.95752 \text { for } m>2 \\
B_{1}= & \frac{-4.0 \eta+3.0 \eta^{2}}{(1-\eta)^{2}}-\frac{9.5}{T^{*}}\left[2.37511 \eta+1.0 \times 10^{-5} \ln (1-\eta)\right] \\
& -\frac{3.37510 \eta-8.69073 \eta^{2}+5.73739 \eta^{3}}{(1-\eta)^{3}}+\frac{288.15745}{\left(T^{*}\right)^{2}}\left[\frac{0.5 \eta^{2}-0.67524 \eta^{3}}{(1+6.75237 \eta)^{3}}\right] \\
B_{2}= & -2.75503 \ln (1-\eta)-\frac{8.21199 \eta-6.30910 \eta^{2}}{(1-\eta)^{2}} \\
& +\frac{12.00320\left(\eta-0.21718 \eta^{2}-4.97058 \eta^{3}+4.78668 \eta^{4}\right)}{T^{*}(1-\eta)^{3}} \\
& +\frac{492.36296\left(\eta^{2}-1.35048 \eta^{3}\right)}{\left(T^{*}\right)^{2}(1+8.26765 \eta)^{3}} \\
\eta & m v^{0} / V \\
T^{*}= & k T / \varepsilon
\end{aligned}
$$

For $i$-component in a fluid mixture, the fugacity coefficient is:

$$
\ln \hat{\phi}_{i}=A_{1}+X_{m} A_{2}+A_{3}+A_{4}+A_{5}-\ln Z
$$

where

$$
\begin{aligned}
& X_{m}=\left(V_{\mathrm{e}, m}-V_{\mathrm{e}, \mathrm{I}}\right) /\left(V_{\mathrm{e}, \mathrm{II}}-V_{\mathrm{e}, \mathrm{I}}\right) \\
& A_{1}=\left(4 \eta-3 \eta^{2}\right) /(1-\eta)^{2}-D_{1} / T^{*}-D_{2} /\left(T^{*}\right)^{2} \\
& A_{2}=2.75503 \ln \frac{1}{1-\eta}+\frac{4.21199 \eta-3.30910 \eta^{2}}{(1-\eta)^{2}}-\frac{D_{3}}{T^{*}}-\frac{D_{4}-D_{2}}{\left(T^{*}\right)^{2}}
\end{aligned}
$$




$$
\begin{aligned}
& A_{3}=m_{i} v_{i}^{0}(Z-1) / m v^{0} \\
& A_{4}=D_{5}\left[-D_{1}-\frac{2 D_{2}}{T^{*}}+X_{m}\left(-D_{3}-\frac{2 D_{4}-2 D_{2}}{T^{*}}\right)\right] \\
& D_{1}=-22.56355 \eta-0.0001 \ln \frac{1}{1-\eta}+\frac{32.06354-82.56198 \eta^{2}+54.50516 \eta^{3}}{(1-\eta)^{3}} \\
& D_{2}=\frac{144.07873 \eta^{2}-194.57496 \eta^{3}}{(1+6.75237 \eta)^{3}} \\
& D_{3}=-34.89251 \eta+0.0001 \ln \frac{1}{1-\eta}+\frac{37.39592 \eta-92.41305 \eta^{2}+58.19954 \eta^{3}}{(1-\eta)^{3}} \\
& D_{4}=\frac{492.36296 \eta^{2}-664.92305 \eta^{3}}{(1+8.26765 \eta)^{3}}
\end{aligned}
$$

For a binary mixture of components $i$ and $j$

$$
D_{5}=\frac{2 s_{i} s_{j}\left(s_{i} \varepsilon_{i i}-s_{i} \varepsilon_{i j}+s_{j} \varepsilon_{i j}-s_{j} \varepsilon_{j j}\right)}{x_{i} k T}
$$

where

$$
s_{k}=\frac{x_{k} m_{k}}{\sum x_{j} m_{j}} \quad k=1,2
$$

\section{References}

[1] W.G. Chapman, K.E. Gubbins, G. Jackson, M. Radosz, Ind. Eng. Chem. Res. 29 (1990) 1709-1721.

[2] A. Yethiraj, K.C. Hall, J. Chem. Phys. 95 (1991) 8494-8506.

[3] C.S. Wu, Y.P. Chen, Fluid Phase Equilibria 101 (1994) 3-26.

[4] C.S. Wu, M. Tang, Y.P. Chen, Fluid Phase Equlibria 117 (1996) 26-32.

[5] N. Kalospiros, D. Tassios, Ind. Eng. Chem. Res. 34 (1995) 2117-2124.

[6] J.L. Liu, D.S.H. Wong, Fluid Phase Equilibria 117 (1996) 92-99.

[7] M.J. Huron, J. Vidal, Fluid Phase Equilibria 3 (1979) 255-271.

[8] T. Oishi, J.M. Prausnitz, Ind. Eng. Chem. Proc. Des. Dev. 17 (1978) 333-339.

[9] I.C. Sanchez, R.H. Lacombe, Macromolecules 11 (1978) 1145-1156.

[10] F. Chen, A. Fredenslund, P. Rasmussen, Ind. Eng. Chem. Res. 29 (1990) 875-882.

[11] R.D. Newman, J.M. Prausnitz, AIChE J. 19 (1973) 704-710.

[12] J.L. Duda, G.K. Kimmerly, W.L. Sigelko, J.S. Vrentas, Ind. Eng. Chem. Fundam. 12 (1973) 133-136.

[13] C.E.H. Bawn, R.F.J. Freeman, A.R. Kamaliddin, Trans. Faraday Soc. 46 (1950) 677-684.

[14] E.C. Baughan, Trans. Faraday Soc. 44 (1948) 495-506.

[15] Y. Iwai, Y. Arai, J. Chem. Eng. Jpn. 22 (1989) 155-160.

[16] W. Hao, H.S. Elbro, P. Alessi, Chemistry Data Series, Vol. XIV, DECHEMA, Frankfurt, 1991.

[17] A. Nakajima, H. Yamakawa, I. Sakurada, J. Polym. Sci. 35 (1959) 489-495.

[18] N.F. Brockmeier, R.W. McCoy, J.A. Meyer, Macromolecules 6 (1973) 176-180.

[19] W.R. Krigbaum, D.O. Geymer, J. Am. Chem. Soc. 81 (1959) 1859-1868. 